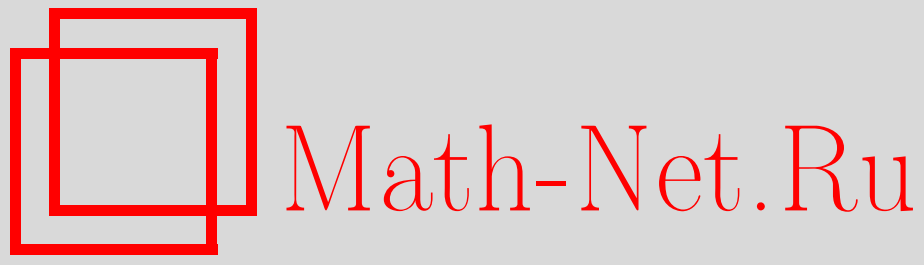

А. Г. Эршлер, О степенях роста конечно порожденных групп, Функи. анализ и его прил., 2005, том 39, выпуск 4, 86-89

DOI: https://doi.org/10.4213/faa90

Использование Общероссийского математического портала Math-Net.Ru подразумевает, что вы прочитали и согласны с пользовательским соглашением

http://www . mathnet.ru/rus/agreement

Параметры загрузки:

IP : 3.91 .87 .62

26 апреля 2023 г., 14:26:49

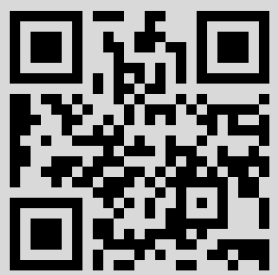


УДК 512.54

\title{
О степенях роста конечно порожденных групп
}

\author{
(C) 2005. А. Г. ЭРшЛЕР
}

Рассмотрим конечно порожденную группу $G$. Пусть $S=\left\{g_{1}, \ldots, g_{m}\right\}$ какая-либо ее конечная система образующих, а $l_{S}$ и $d_{S}-$ словарная длина и словарная метрика, соответствующие $S$. Напомним, что функция роста группы $G$ по отношению к $S$ - это

$$
v_{G, S}(n)=\#\left\{g \in G: l_{S}(g) \leqslant n\right\} .
$$

Если $S_{1}$ и $S_{2}$ - две системы образующих группы $G$, то существуют $K_{1}, K_{2}>0$, такие, что для любого $n$ выполнены неравенства $v_{G, S_{1}}(n) \leqslant v_{G, S_{2}}\left(K_{2} n\right)+K_{2}$ и $v_{G, S_{2}}(n) \leqslant v_{G, S_{1}}\left(K_{1} n\right)+K_{1}$. Говорят, что группа $G$ имеет полиномиальный рост, если для некоторых $A, d>0$ и любого натурального $n$ выполняется неравенство $v_{G, S}(n) \leqslant A n^{d}$. Группа $G$ называется группой экспоненциального роста если $v_{G, S}(n) \geqslant C^{n}$ для некоторого $C>1$. Ясно, что $v_{G, S}(n) \leqslant(n+1) 2 m(2 m-1)^{n-1}$ для любых $G, S$. Также ясно, что свойство иметь полиномиальный или экспоненциальный рост не зависит от выбранной системы образующих. Говорят, что группа имеет промежуточный рост, если ее рост не является ни экспоненциальным, ни полиномиальным. Известно, что группа имеет полиномиальный рост тогда и только тогда, когда она виртуально нильпотентна [7], и что рост любой разрешимой или линейной группы либо полиномиальный, либо экспоненциальный (см. [9, 12,11]). Первые примеры групп промежуточного роста построены Р. И. Григорчуком в [3]. (Обсуждение общих вопросов, связанных с ростом, можно найти в [6] и [8]. Некоторые новые оценки роста групп Григорчука недавно были получены в [1].)

Ниже мы напомним одну из конструкций работы [3]. Для любого $i \geqslant 1$ фиксируем некоторую биекцию $m_{i}:(0,1] \rightarrow(0,1]$. Рассмотрим элемент $g$, который действует на $(0,1]$ следующим образом. На $\left(0, \frac{1}{2}\right]$ он действует, как $m_{1}$ на $(0,1]$, на $\left(\frac{1}{2}, \frac{3}{4}\right]$ - как $m_{2}$ на $(0,1]$, на $\left(\frac{3}{4}, \frac{7}{8}\right]-$ как $m_{3}$ на $(0,1]$, и т. д. Более точно, возьмем $r \geqslant 1$, и пусть $\Delta_{r}=\left(1-2^{-(r-1)}, 1-2^{-r}\right]$. Рассмотрим аффинное отображение $\alpha_{r}$ из $\Delta_{r}$ на $(0,1]$. Отображение $g:(0,1] \rightarrow(0,1]$ определено формулой

$$
g(x)=\alpha_{r}^{-1}\left(m_{r}\left(\alpha_{r}(x)\right)\right)
$$

для $x \in \Delta_{r}$. В этой ситуации мы будем писать

$$
g=m_{1}, m_{2}, m_{3}, \ldots .
$$

Пусть $a$ - циклическая перестановка полуинтервалов отрезка $(0,1]$, т. е.

$$
a(x)=x+\frac{1}{2} \quad \text { при } x \in\left(0, \frac{1}{2}\right] \quad \text { и } \quad a(x)=x-\frac{1}{2} \quad \text { при } x \in\left(\frac{1}{2}, 1\right] .
$$

Пусть $P=a$ и $T$ - тождественное отображение отрезка $(0,1]$. (Здесь и для $b$ и $d$, определяемых ниже, мы следуем обозначениям из [3].)

Рассмотрим последовательность натуральных чисел $s=\left\{s_{1}, s_{2}, s_{3}, \ldots\right\}$ и положим

$$
w(s)=\underbrace{P P P \ldots P P}_{s_{1}} P T \underbrace{P P P \ldots P P}_{s_{2}} P T \underbrace{P P P \ldots P P}_{s_{3}} P T \ldots
$$


И

$$
u(s)=\underbrace{P P P \ldots P P}_{s_{1}} T P \underbrace{P P P \ldots P P}_{s_{2}} T P \underbrace{P P P \ldots P P}_{s_{3}} T P \ldots
$$

Пусть $b(s)$ действует на $(0,1]$, как $w(s)$, а $c(s)$ - как $u(s)$, и пусть $G(s)-$ это группа с системой образующих $S(s)=\{a, b(s), c(s)\}$. Группы $G(s)$ являются частным случаем конструкции из [3], где доказано, что для любой субэкспоненциально растущей функции $\rho$ существует последовательность $s$, такая, что функция роста $v_{G(s), S(s)}(n)$ больше, чем $\rho(n)$, для бесконечно большого количества чисел $n$. В этой заметке мы докажем следующее:

ТЕОрема 1. Для любой функиии $\rho$, такой, что $\rho(n)=o(\exp (\epsilon n))(n p u n \rightarrow$ $\infty)$ для любого $\epsilon>0$, существует группа $H$ промежуточного роста, такая, чmo

$$
v_{H, S_{H}}(n) \geqslant \rho(n)
$$

для некоторой системы образующих $S_{H}$ группь $H$ и для любого $n>0$.

Ясно, что для доказательства теоремы достаточно построить группу $H_{1}$, такую, что неравенство в формулировке теоремы выполнено для всех достаточно больших $n$ (после чего в качестве $H$ можно взять прямое произведение группы $H_{1}$ с достаточно большой конечной (или свободной абелевой) группой).

Мы покажем, что существуют последовательности $s$ и $r$ (зависящие от $\rho$ ), такие, что в качестве $H_{1}$ можно взять прямое произведение $G(s) \times G(r)$. Для $G(s)$ и $G(r)$ мы оценим рост, используя тщательный анализ аргумента из [3].

Существование полугрупп сколь угодно большого промежуточного роста уже было известно [10].

ЗАмечАния. 1) Группа $H_{1}=G(r) \times G(s)$ есть 2-группа, т. е. для любого $h \in H$ существует $d \in \mathbb{Z}$, такое, что $h^{2^{d}}=e($ так как $G(r), G(s)$ являются 2-группами, см. [3]).

2) Группу $H$ можно выбрать без кручения. Для этого вместо $G(s)$ и $G(r)$ надо рассмотреть построенные в [4] группы промежуточного роста без кручения $G^{\prime}(s)$ и $G^{\prime}(r)$, такие, что $G(s)$ и $G(r)$ являются факторгруппами групп $G^{\prime}(s)$ и $G^{\prime}(r)$ соответственно.

3) Для любого простого $p$ группу $H$ можно выбрать $p$-группой. Для этого вместо $G(s)$ и $G(r)$ можно рассмотреть построенные в [4] $p$-группы промежуТОчНого роста.

ДокАЗАТЕЛЬСтво ТЕОРЕмы 1. Последовательности $\left\{s_{i}\right\}$ и $\left\{r_{i}\right\}$ будут построены одновременной индукцией по $i$ таким образом, что $r_{1}<s_{1}<r_{2}<s_{2}<$ $r_{3}<\cdots$. Напомним еще одну конструкцию из [3]: для любых $r_{1}, \ldots, r_{k}$ в [3] построена группа $\widetilde{G}\left(r_{1}, \ldots r_{k}\right)$ со следующими свойствами:

- $\widetilde{G}\left(r_{1}, \ldots, r_{k}\right)$ - виртуально разрешимая 3-порожденная группа; ее образующие мы обозначим через $a, b, c$,

- для любых натуральных $r_{k+1}, r_{k+2}, \ldots$ шары в $G(r)$, где $r=\left\{r_{1}, \ldots, r_{k}\right.$, $\left.r_{k+1}, \ldots\right\}$, и $G\left(r_{1}, \ldots, r_{k}\right)$ радиуса $\left[\frac{1}{2} 2^{r_{1}+\cdots+r_{k+1}+2 k}\right]$ изометричны. В частности, шары радиуса $r_{k+1}$ в этих группах изометричны. Мы рассматриваем словарную метрику в $\widetilde{G}\left(r_{1}, \ldots, r_{k}\right)$, соответствующую $S=\{a, b, c, d=b c\}$, а в $G(r)$ - соответствующую $S(r)=\{a, b(r), c(r), d(r)=b(r) c(r)\}$. (Наши обозначения отличаются от обозначений из [3], где $\widetilde{G}\left(r_{1}, \ldots, r_{k}\right)$ определены в более общем 
контексте и обозначены через $\widetilde{G}(\omega)$ для последовательностей $\omega=\left\{\omega_{i}\right\}$ из $0,1,2$, постоянных с некоторого места.)

Пусть $c\left(r_{1}, \ldots, r_{k}\right)$ - экспоненциальный показатель роста для $\left(\widetilde{G}\left(r_{1}, \ldots, r_{k}\right), S\right)$, T. e.

$$
c\left(r_{1}, \ldots, r_{k}\right)=\lim _{n \rightarrow \infty} \sqrt[n]{v_{\widetilde{G}\left(r_{1}, \ldots, r_{k}\right), S}(n)} .
$$

По хорошо известному свойству роста (см., например, [8]) для любого $n \geqslant 1$

$$
v_{\widetilde{G}\left(r_{1}, \ldots, r_{k}\right), S}(n) \geqslant c\left(r_{1}, \ldots, r_{k}\right)^{n} \text {. }
$$

Следовательно, для любых $r_{k+1}, r_{k+2}, \ldots$ и $1 \leqslant n \leqslant r_{k+1}$

$$
v_{G(r), S(r)}(n) \geqslant c\left(r_{1}, \ldots, r_{k}\right)^{n} \text {. }
$$

Положим $r_{1}=1, s_{1}=2$. Существует $n_{1}=N$, такое, что для любого $n \geqslant n_{1}$

$$
c\left(s_{1}\right)^{n} \geqslant \rho(n) \text {. }
$$

Возьмем любое $r_{2}$, для которого $r_{2} \geqslant n_{1}, r_{2}>s_{2}$. Существует $m_{1}>r_{2}$, такое, что для любого $n \geqslant m_{1}$

$$
c\left(r_{1}, r_{2}\right)^{n} \geqslant \rho(n) .
$$

Положим $s_{2}=m_{1}$ и рассмотрим $n_{2}>s_{2}$, такое, что для любого $n \geqslant n_{2}$

$$
c\left(s_{1}, s_{2}\right)^{n} \geqslant \rho(n) .
$$

Положим $r_{3}=n_{2}$ и так далее. В результате мы получим последовательности $s$ и $r$, такие, что $r_{1}<s_{1}<r_{2}<s_{2}<r_{3}<\cdots$. По построению для любого $n$, $r_{i+1} \leqslant n<s_{i+1}$,

$$
\rho(n) \leqslant v_{\widetilde{G}\left(s_{i}\right)}(n)=v_{G(s)}(n) \leqslant v_{H_{1}, S_{H_{1}}}(n)
$$

и для любого $n, s_{i+1} \leqslant n<r_{i+2}$,

$$
\rho(n) \leqslant v_{\widetilde{G}\left(r_{i+1}\right)}(n)=v_{G(r)}(n) \leqslant v_{H_{1}, S_{H_{1}}}(n) .
$$

(В $H_{1}=G(r) \times G(s)$ мы рассматриваем систему образующих $S_{H_{1}}$, являющуюся объединением образующих групп $G(r)$ и $G(s)$.

Обобщения. Пусть $U_{2}$ - группа, порожденная $a$ и всеми элементами $g$ вида $g=$ PТРРТРТ... (для всех возможных выборов последовательностей из $P$ и Т).

ПРЕДЛОЖЕНИЕ. 1) Любая конечно порожденная подгруппа группь $U_{2}$ uмеет субэкспоненииальный рост.

2) Для любой функиии $\rho$, такой, что $\rho(n)=o(\exp (\epsilon n))(n p u n \rightarrow \infty)$ для любого $\epsilon>0$, существует подгруппа $\mathrm{H}$ группъ $U_{2}$, такая, что

$$
v_{H, S_{H}}(n) \geqslant \rho(n)
$$

для некоторой системы $S_{H}$ образующих группы $H$ и любого $n>0$.

Доказательство второго утверждения предложения аналогично доказательству теоремы 1. Первое утверждение предложения доказывается при помощи стандартных аргументов из [3], и мы опускаем доказательство.

Приведем без доказательства еще одно обобщение теоремы 1.

Обозначим через $I_{s}(\psi)$ класс конечно порожденных групп $G$, функция роста которых удовлетворяет неравенству

$$
v_{G, S}(n) \leqslant A(\psi(B n))
$$


для бесконечно многих $n$ (и некоторой системы образующих $S$, причем константы $A$ и $B$ зависят от $S)$.

Tеорема 1а. Cуществует $\alpha<1$, такое, что для любой функиии $\rho$ удовлетворяющей условию $\rho(n)=o(\exp (\epsilon n))(n p u n \rightarrow \infty)$ для любого $\epsilon>0$, существует группа $H=H_{1} \times H_{2}$, такал, что $H_{1}, H_{2} \in I_{s}\left(\exp \left(n^{\alpha}\right)\right) u$

$$
v_{H, S_{H}}(n) \geqslant \rho(n)
$$

для всех $n>0$.

Автор выражает благодарность Р. И. Григорчуку за внимательное прочтение предварительной версии этой заметки.

\section{ЛитератУРА}

1. Erschler A. Boundary behavior for groups of subexponential growth. Ann. Math., 160, No. 3, 1183-1210 (2004). 2. Григорчук P. И. Функц. анализ и его прил., 14, 41-43 (1980). 3. Григорчук Р. И. Изв. АН СССР, 25, №2, 259-300 (1985). 4. Григорчук Р. И. Матем. сб., 54, 185-205 (1986). 5. Григорчук Р. И. Группы промежуточного роста и их приложения. Докт. дисс., 1985. 6. Григорчук Р. И., Курчанов П. Ф. Итоги науки и техники. Современные проблемы математики, Фундаментальные направления, т. 58, ВИНИТИ, М., 1990, с. 191-256. 7. Gromov M. Inst. Hautes Études Sci. Publ. Math., 53, 53-73 (1981). 8. de la Harpe P. Topics in Geometric Group Theory, The University of Chicago Press, 2000. 9. Milnor J. J. Differential Geometry, 2, 447-449 (1968). 10. Shneyerson L. Сообщение на конференции "International Conference on Group Theory: Combinatorial, Geometric, and Dynamical Aspects of Infinite Groups", Гаета, 1-6 июня 2003. 11. Tits J. J. Algebra, 20, 250-270 (1979). 12. Wolf J. A. J. Differential Geometry, 2, 421-446 (1968).

CNRS, Université Lille 1, UFR de Mathématiques email: erschler@pdmi.ras.ru, Anna.Erschler@math.univ-lille1.fr
Поступило в редакцию 7 февраля 2004 г.

\section{Точное значение коэффициентов нормальной структуры и WCS-коэффициентов в классе функциональных пространств Орлича}

(c) 2005. Янь ЦЯн

Коэффициент нормальной структуры $N(X)$ банахова пространства $X$ определяется формулой [1]

$$
N(X)=\inf \left\{\frac{d(B)}{r(B)}: B \subset X \text { ограничено, замкнуто и выпукло, } d(B)>0\right\},
$$

где $d(B)=\sup \{\|x-y\|: x, y \in B\}$ - диаметр, а $r(B)$ - относительный чебышёвский радиус множества $B$, т. е. $r(B)=\inf \{\sup \{\|x-y\|: x \in B\}: y \in B\}$.

Для банахова пространства $X$, не обладающего свойством Шура, коэффициент слабо сходящихся последовательностей ( $W C S$-коэффициент) определяется 